\title{
THE INTEGRAL OF A FUNCTION WITH RESPECT TO A FUNCTION. II
}

\section{RALPH E. LANE}

1. Introduction. If $x$ is a function, ${ }^{1} y$ is a function, and $G$ is a realnumber set, then by the graph, $(x, y, G)$, of $y$ with respect to $x$ in $G$ we mean the image of $G$ under a transformation $T$ such that if $p$ is in $G$, then $T(p)$ is the ordered number-pair $x(p), y(p)$.

Consider the following problem in integration. Let $U$ denote the set such that $s$ is a member of $U$ if and only if $s$ is the graph of a function with respect to a function in an interval. We are to select a subset $X$ of $U$ and assign to each member $(v, u,[a, b])$ of $X$ a number $\int_{a}^{b} u(x) d v(x)$ so that the following statements are true.

(1.1) If $(v, u,[a, b])$ is in $U$ and $u(x)=0$ for each number $x$ in $[a, b]$, then $(v, u,[a, b])$ is in $X$ and $\int_{a}^{b} u(x) d v(x)=0$.

(1.2) Suppose that $(v, u,[a, b])$ is in $X$, and $a_{11}, a_{12}, a_{13}, a_{21}, a_{22}, a_{23}$ is a number-sequence, and $f(x)=a_{11} u(x)+a_{12} v(x)+a_{13}$ and $g(x)$ $=a_{21} u(x)+a_{22} v(x)+a_{23}$ for each number $x$ in $[a, b]$. Then $(g, f,[a, b])$ is in $X$, and

$$
\begin{aligned}
& \int_{a}^{b} f(x) d g(x)-2^{-1}[f(a)+f(b)][g(b)-g(a)] \\
& =\left(a_{11} a_{22}-a_{12} a_{21}\right)\left\{\int_{a}^{b} u(x) d v(x)-2^{-1}[u(a)+u(b)][v(b)-v(a)]\right\} .
\end{aligned}
$$

(1.3) Suppose that $(v, u,[a, b])$ is in $U$ and that $a<c<b$. Then $(v, u,[a, b])$ is in $X$ if and only if it is true that $(v, u,[a, c])$ and $(v, u,[c, b])$ are in $X$; moreover, if $(v, u,[a, b])$ is in $X$, then $\int_{a}^{b} u(x) d v(x)=\int_{a}^{c} u(x) d v(x)+\int_{c}^{b} u(x) d v(x)$.

In passing, we remark that if $u$ is a step-function, $v$ is a stepfunction, and $[a, b]$ is an interval, then $(v, u,[a, b])$ is in $X$ and the number $\int_{a}^{b} u(x) d v(x)$ is specified by (1.1), (1.2), and (1.3).

One solution of this integration problem has been given in [2], [3], and [1], as may be verified from Definition 2.1 and Theorem 2.1 of [1]. Now from Definition 2.1 of [1] it can readily be seen that if $u$ is integrable with respect to $v$ in $[a, b]$, then there is a countable subset $G$ of $[a, b]$ such that the following statement is true: if $\epsilon$ is a posi-

Presented to the Society, November 28, 1953; received by the editors October 23, 1953 and, in revised form, July 22, 1954.

1 We use the notation and terminology of [1]; in particular, the words "function," "step-function," "interval," "subdivision," "refinement," and "integrable" are used as in [1]. 
tive number, then there is a subdivision $D$ of $[a, b]$, each of whose terms is in $G$, such that $\left|S_{E}(u, v)-\int_{a}^{b} u(x) d v(x)\right|<\epsilon$ if $E$ is a refinement of $D$ each of whose terms is in $G$.

This fact suggests the possibility of a new way of defining an integral as a limit of approximating sums (see $\$ 2$ of this paper) so as to retain the properties (1.1), (1.2), (1.3). Most of the fundamental ideas involved are illustrated by the following example, in which we consider the integral of a totally discontinuous function with respect to a totally discontinuous function.

EXAMPLE 1.1. Suppose that $[a, b]$ is an interval, $c$ is a positive number, $u(x)=c$ and $v(x)=x$ if $x$ is an irrational number, and $c+1$ $\leqq u(x) \leqq c+10$ and $x+1 \leqq v(x) \leqq x+10$ if $x$ is a rational number. Let $G$ denote the set whose members are $a, b$, and the irrational numbers between $a$ and $b$; and let $H$ denote the set whose members are the rational numbers between $a$ and $b$. Then

(i) the graph $(v, u, H)$ is a singular graph (Definition 2.1),

(ii) $G$ is a summability set (Definition 2.3) for $u$ and $v$ in $[a, b]$,

(iii) $\int_{a}^{b} u(x) d v(x)=c(b-a)+2^{-1}[c+u(b)][v(b)-b]-2^{-1}[u(a)+c]$ $\cdot[v(a)-a]$, and

(iv) if $A$ is a subdivision of $[a, b]$ and $\epsilon$ is a positive number, then there is a refinement $B$ of $A$ such that $\left|S_{C}(u, v)-\int_{a}^{b} u(x) d v(x)\right|<\epsilon$ if $C$ is a refinement of $B$ each of whose terms is a term of $B$ or a number in $G$.

2. Definitions and lemmas. We now introduce three definitions upon which the rest of this paper will be based.

Definition 2.1. If $u$ is a function, $v$ is a function, and $H$ is a realnumber set, then the statement that $(v, u, H)$ is a singular graph means that if $\epsilon$ is a positive number, then there is a countable set $I$ of intervals such that

(i) if $[c, d]$ is in $I$, then neither $c$ nor $d$ is in $H$,

(ii) if $x$ is in $H$, then $x$ is in an interval of $I$, and

(iii) if $\left[a_{p}, b_{p}\right], p=1,2,3, \cdots$, are the members of $I$, and $A_{p}$ and $B_{p}$ are subdivisions of $\left[a_{p}, b_{p}\right]$, then $\sum_{(p)}\left|S_{A_{p}}(u, v)-S_{B_{p}}(u, v)\right|<\epsilon$.

EXAmple 2.1. Suppose that $u(x)=0$ or 1 , according as $x$ is a rational number or an irrational number, and that $v(x)=1+x$ or $x$, according as $x$ is a rational number or an irrational number. Let $[a, b]$ denote an interval such that $a$ and $b$ are irrational numbers, and let $H$ denote the set of all rational numbers between $a$ and $b$. Then $(v, u, H)$ is a singular graph.

REMARK 2.1. If $D$ and $E$ are subdivisions of $[a, b]$, then $S_{D}(u, v)$ $+S_{D}(v, u)=u(b) v(b)-u(a) v(a)=S_{E}(u, v)+S_{E}(v, u)$, so that $S_{D}(v, u)$ 
$-S_{E}(v, u)=S_{E}(u, v)-S_{D}(u, v)$. Hence if $(v, u, H)$ is a singular graph, then so is $(u, v, H)$.

Definition 2.2. The statement that $x$ is an exceptional number for the functions $u$ and $v$ in the interval $[a, b]$ means that there is a subinterval $[c, d]$ of $[a, b]$ containing $x$ such that if $[p, q]$ is a subinterval of $[c, d]$ then

(i) $u$ is integrable with respect to $v$ in $[p, q]$ if $x$ is not in $[p, q]$,

(ii) $u$ is not integrable with respect to $v$ in $[p, q]$ if $x$ is in $[p, q]$.

EXAMPLE 2.2. Suppose that $u$ is a function such that if $x$ is a real number other than 1 , then the limits $u(x+)$ and $u(x-)$ exist, but the limit $u(1+)$ does not exist. Suppose that $v$ is a function of bounded variation such that $v(1+) \neq v(1)$. Then 1 is an exceptional number for $u$ and $v$ in $[0,2]$.

Definition 2.3. The statement that $G$ is a summability set for the functions $u$ and $v$ in the interval $[a, b]$ means that

(i) $G$ is a subset of $[a, b]$, and $a$ and $b$ are in $G$, and none of the numbers in $G$ is an exceptional number for $u$ and $v$ in $[a, b]$,

(ii) if $\epsilon$ is a positive number, then there is a subdivision $D$ of $[a, b]$, each of whose terms is in $G$, such that if $E$ is a refinement of $G$ each of whose terms is in $G$, then $\left|S_{D}(u, v)-S_{E}(u, v)\right|<\epsilon$, and

(iii) if $G$ is not $[a, b]$ and $H$ is the complement of $G$ in $[a, b]$, then $(v, u, H)$ is a singular graph.

EXAMPLE 2.3. Let $G$ denote the complement in $[a, b]$ of the set $H$ defined in Example 2.1, and let $u$ and $v$ be defined as in Example 2.1. Then $G$ is a summability set for $u$ and $v$ in $[a, b]$; moreover, if $\epsilon$ is a positive number, then there is a subdivision $D$ of $[a, b]$, each of whose terms is in $G$, such that if $E$ is a refinement of $D$ each of whose terms is in $G$, then $\left|S_{E}(u, v)-(b-a)\right|<\epsilon$.

LEMмA 2.1. If $G$ is a summability set for $u$ and $v$ in $[a, b]$, then there is just one number $k$ such that the following statement is true:

(2.1) If $\epsilon$ is a positive number, then there is a subdivision $D$ of $[a, b]$, each of whose terms is in $G$, such that if $E$ is a refinement of $D$ each of whose terms is in $G$ then $\left|S_{E}(u, v)-k\right|<\epsilon$.

Proof. A. Let $D_{1}$ denote a subdivision of $[a, b]$, each of whose terms is in $G$, such that $\left|S_{D_{1}}(u, v)-S_{E}(u, v)\right|<1 / 2$ if $E$ is a refinement of $D_{1}$ each of whose terms is in $G$. For each integer $n$ greater than 1 , let $D_{n}$ denote a refinement of $D_{n-1}$, each of whose terms is in $G$, such that $\left|S_{D_{n}}(u, v)-S_{E}(u, v)\right|<1 / 2^{n}$ if $E$ is a refinement of $D_{n}$ each of whose terms is in $G$. Now if $m$ is a positive integer and $n$ is an integer greater than $m$, then $\left|S_{D_{m}}(u, v)-S_{D_{n}}(u, v)\right|<1 / 2^{m}$; by Cauchy's convergence criterion, there is a number $k$ such that if $n$ 
is a positive integer then $\left|S_{D_{n}}(u, v)-k\right| \leqq 1 / 2^{n}$ and therefore $\left|S_{E}(u, v)-k\right|<1 / 2^{n-1}$ if $E$ is a refinement of $D_{n}$ each of whose terms is in $G$. Hence there is a number $k$ such that $(2.1)$ is true.

B. For $i=1,2$, suppose that $k_{i}$ is a number such that if $k$ is $k_{i}$ then (2.1) is true. Let $\epsilon$ denote a positive number, and for $i=1,2$, let $A_{i}$ denote a subdivision of $[a, b]$, each of whose terms is in $G$, such that if $E$ is a refinement of $A_{i}$ each of whose terms is in $G$ then $\mid S_{E}(u, v)$ $-k_{i} \mid<\epsilon$. Let $E$ denote the refinement of $A_{1}$ whose terms are the terms of $A_{1}$ and $A_{2}$. Then $\left|S_{E}(u, v)-k_{1}\right|<\epsilon$, and $\left|S_{E}(u, v)-k_{2}\right|<\epsilon$, and therefore $\left|k_{1}-k_{2}\right|<2 \epsilon$ if $\epsilon$ is a positive number. Hence $k_{1}=k_{2}$. This completes the proof.

Lemma 2.2. Suppose that $[a, b]$ is an interval, $u$ is a function, $v$ is a function, $\epsilon$ is a positive number, and $\left\{\left[c_{p}, d_{p}\right]\right\}_{p=1}^{n}$ is a finite sequence of intervals such that

(i) if $x$ is in $[a, b]$ then $x$ is in one of the intervals $\left[c_{p}, d_{p}\right]$, and

(ii) if $C_{p}$ and $D_{p}$ are subdivisions of $\left[c_{p}, d_{p}\right], p=1,2, \cdots, n$, then $\sum_{p=1}^{n}\left|S_{C_{p}}(u, v)-S_{D_{p}}(u, v)\right|<\epsilon$.

If $A$ is a subdivision of $[a, b]$ among whose terms are the numbers $c_{p}$ and $d_{p}$ (if any) which are in $[a, b]$, and $B$ is a refinement of $A$, then $\left|S_{A}(u, v)-S_{B}(u, v)\right|<\epsilon$.

Proof. Case $\mathrm{I} ; n=1$. In this case, $[a, b]$ is a subinterval of $\left[c_{1}, d_{1}\right]$. Let $A$ denote a subdivision of $[a, b]$, and let $B$ denote a refinement of $A$. Let $C_{1}$ and $D_{1}$ denote the subdivisions of $\left[c_{1}, d_{1}\right]$ whose terms are $c_{1}, d_{1}$, and the terms of $A$ and $B$, respectively. Then $S_{A}(u, v)-S_{B}(u, v)$ $=S_{C_{1}}(u, v)-S_{D_{1}}(u, v)$, and hence $\left|S_{A}(u, v)-S_{B}(u, v)\right|<\epsilon$.

Case II $; n>1$. Let $K$ denote the number sequence $c_{1}, d_{1}, c_{2}, d_{2}$, $\cdots, c_{n} ; d_{n}$. Let $A$ denote a subdivision of $[a, b]$ among whose terms are the terms (if any) of $K$ which are in $[a, b]$, and let $B$ denote a refinement of $A$. Let $C_{1}$ denote the subdivision of $\left[c_{1}, d_{1}\right]$ whose terms are the terms of $A$ and $K$ which are in $\left[c_{1}, d_{1}\right]$, and let $D_{1}$ denote the subdivision of $\left[c_{1}, d_{1}\right]$ whose terms are the terms of $B$ and $K$ which are in $\left[c_{1}, d_{1}\right]$. For $p=2,3, \cdots, n$, let $C_{p}$ denote the subdivision of $\left[c_{p}, d_{p}\right]$ whose terms are the terms of $K$ which are in $\left[c_{p}, d_{p}\right]$ and the terms (if any) of $A$ which are in $\left[c_{p}, d_{p}\right]$ but are not terms of $K, C_{1}, C_{2}, \cdots, C_{p-1}$; and let $D_{p}$ denote the subdivision of $\left[c_{p}, d_{p}\right]$ whose terms are the terms of $K$ which are in $\left[c_{p}, d_{p}\right]$ and the terms (if any) of $B$ which are in $\left[c_{p}, d_{p}\right]$ but are not terms of $K, D_{1}, D_{2}, \cdots, D_{p-1}$. Then $S_{A}(u, v)-S_{B}(u, v)=\sum_{p=1}^{n}\left[S_{C_{p}}(u, v)\right.$ $\left.-S_{D_{p}}(u, v)\right] ;$ so $\left|S_{A}(u, v)-S_{B}(u, v)\right| \leqq \sum_{p=1}^{n}\left|S_{C_{p}}(u, v)-S_{D_{p}}(u, v)\right|$ $<\epsilon$. This completes the proof.

Lemma 2.3. Suppose that $G_{1}$ and $G_{2}$ are summability sets for $u$ and $z$ 
in $[a, b]$. If there is a subinterval $[c, d]$ of $[a, b]$ such that none of the numbers between $c$ and $d$ is in $G_{1}$ and in $G_{2}$, then $u$ is integrable with respect to $v$ in $[c, d]$.

Proof. Case I; none of the numbers between $c$ and $d$ is in $G_{2}$. Since $G_{2}$ is a summability set for $u$ and $v$ in $[a, b]$, it follows that if $\epsilon>0$ then there is an interval $\left[c_{1}, d_{1}\right]$, of which $[c, d]$ is a subinterval, such that $c_{1}$ and $d_{1}$ are in $G_{2}$ and $\left|S_{C}(u, v)-S_{D}(u, v)\right|<\epsilon$ if $C$ and $D$ are subdivisions of $\left[c_{1}, d_{1}\right]$. By Lemma 2.2 , if $A$ is a subdivision of $[c, d]$ and $B$ is a refinement of $A$, then $\left|S_{A}(u, v)-S_{B}(u, v)\right|<\epsilon$. Hence $u$ is integrable with respect to $v$ in $[c, d]$.

Case II; none of the numbers between $c$ and $d$ is in $G_{1}$. For this case, the argument is similar to that used in Case I.

Case III; there are between $c$ and $d$ a number which is in $G_{1}$ and a number which is in $G_{2}$. Let $\epsilon$ denote a positive number. For $i=1,2$, let $I_{i}$ denote a countable set (see Definition 2.1) of subintervals of $[a, b]$ such that

(1) if $[r, s]$ is in $I_{i}$, then $r$ and $s$ are in $G_{i}$,

(2) if $x$ is between $a$ and $b$ but not in $G_{i}$, then there is an interval $[r, s]$ in $I_{i}$ such that $r<x<s$, and

(3) if the members of $I_{i}$ are $\left[a_{p}, b_{p}\right]$, and $A_{p}$ and $B_{p}$ are subdivisions of $\left[a_{p}, b_{p}\right], p=1,2, \cdots$, then $\sum_{(p)}\left|S_{A_{p}}(u, v)-S_{B_{p}}(u, v)\right|<\epsilon / 2$.

Now let $[h, k]$ denote a subinterval of $[c, d]$ such that if $x$ is in $[h, k]$ then $x$ is not in $G_{1}$ or $x$ is not in $G_{2}$. Then $[h, k]$ can be covered by a set $s_{0}$ of segments $\left(a_{p}, b_{p}\right)$ such that the intervals $\left[a_{p}, b_{p}\right]$ are from $I_{1}$ and $I_{2}$. Let $\left[c_{p}, d_{p}\right], p=1,2, \cdots, n$, denote a finite subset of $s_{0}$ such that the segments $\left(c_{p}, d_{p}\right)$ cover $[h, k]$. Let $D$ denote the subdivision of $[h, k]$ whose terms are the numbers $h$ and $k$ and the numbers $c_{p}$ and $d_{p}$ which are in $[h, k]$. By Lemma 2.2, if $E$ is a refinement of $D$, then $\left|S_{D}(u, v)-S_{E}(u, v)\right|<\epsilon$; so $u$ is integrable with respect to $v$ in $[h, k]$. Now either $u$ is integrable with respect to $v$ in $[c, k]$ or $c$ is an exceptional number for $u$ and $v$ in $[a, b]$ and is not in $G_{1}$ or $G_{2}$, in which case $u$ is integrable with respect to $v$ in $[c, k]$ by the above argument; similarly, if $k<d$, then $u$ is integrable with respect to $v$ in $[k, d]$ and consequently in $[c, d]$. This completes the proof.

LEMmA 2.4. Suppose that $G_{1}$ and $G_{2}$ are summability sets for $u$ and $v$ in $[a, b]$ and that $x$ is a number between $a$ and $b$ which is in $G_{2}$ but not in $G_{1}$. If $\delta$ is a positive number, then there is a subinterval $[c, d]$ of $[a, b]$ such that

(i) each of $c$ and $d$ is in $G_{1}$ and in $G_{2}$,

(ii) $c<x<d$, and 
(iii) there is a subdivision $D$ of $[c, d]$ such that if $E$ is a refinement of $D$ then $\left|S_{D}(u, v)-S_{B}(u, v)\right|<\delta$.

Proof. Case I; $G_{2}$ is $[a, b]$. Let $H$ denote the complement of $G_{1}$ in $[a, b]$; then $x$ is in $H$, and $(v, u, H)$ is a singular graph. Hence if $\delta$ is a positive number, then there is a subinterval $[c, d]$ of $[a, b]$ such that $c$ and $d$ are in $G_{1}$ and in $G_{2}, x$ is between $c$ and $d$, and $\mid S_{D}(u, v)$ $-S_{E}(u, v) \mid<\delta$ if $D$ and $E$ are subdivisions of $[c, d]$.

Case II; $G_{2}$ is a proper subset of $[a, b]$. Suppose that $\delta$ is a positive number and that $\epsilon=\delta / 2$. Let sets $I_{1}$ and $I_{2}$ be selected as in the proof of Lemma 2.3. Let $\left[c_{1}, d_{1}\right]$ denote an interval from $I_{1}$ such that $c_{1}<x<d_{1}$. If there is a number $y$ in $\left[c_{1}, x\right]$ which is in $G_{1}$ and in $G_{2}$, let $c$ denote such a number $y$, and let $D_{1}$ denote a subdivision of $[c, x]$; if $E$ is a refinement of $D_{1}$, then $\left|S_{D_{1}}(u, v)-S_{E}(u, v)\right|<\epsilon$. If none of the numbers in $\left[c_{1}, x\right]$ is in $G_{1}$ and in $G_{2}$, let $c^{\prime}$ denote the smallest number $t$ such that if $y$ is a number in $[a, x]$ which is in $G_{1}$ and in $G_{2}$ then $y \leqq t$. If $c^{\prime}$ is in $G_{1}$ and in $G_{2}$, let $c$ denote $c^{\prime}$; by Lemma 2.3, $u$ is integrable with respect to $v$ in $[c, x]$, and hence there is a subdivision $D_{1}$ of $[c, x]$ such that $\left|S_{D_{1}}(u, v)-S_{E}(u, v)\right|<\epsilon$ if $E$ is a refinement of $D_{1}$. If $c^{\prime}$ is not in $G_{1}$ and in $G_{2}$, let $\left[c^{\prime \prime}, d^{\prime \prime}\right]$ denote an interval from $I_{1}$ or $I_{2}$ such that $c^{\prime \prime}<c^{\prime}<d^{\prime \prime}$, and let $c$ denote a number in $\left[c^{\prime \prime}, c^{\prime}\right]$ which is in $G_{1}$ and in $G_{2}$; by Lemma $2.3, u$ is integrable with respect to $v$ in $\left[c^{\prime}, x\right]$, and by Lemma 2.2 if $A$ is a subdivision of $\left[c, c^{\prime}\right]$ and $B$ is a refinement of $A$ then $\left|S_{A}(u, v)-S_{B}(u, v)\right|<\epsilon / 2$; hence there is a subdivision $D_{1}$ of $[c, x]$ such that $\left|S_{D_{1}}(u, v)-S_{E}(u, v)\right|$ $<\epsilon$ if $E$ is a refinement of $D_{1}$.

Similarly we find a number $d$ in $[x, b]$ which is in $G_{1}$ and in $G_{2}$, and a subdivision $D_{2}$ of $[x, d]$ such that $\left|S_{D_{2}}(u, v)-S_{E}(u, v)\right|<\epsilon$ if $E$ if a refinement of $D_{2}$. Let $D$ denote the subdivision of $[c, d]$ whose terms are the terms of $D_{1}$ and $D_{2}$; if $E$ is a refinement of $D$, then $\left|S_{D}(u, v)-S_{E}(u, v)\right|<2 \epsilon=\delta$. This completes the proof.

Lemma 2.5. Suppose that for $i=1,2, G_{i}$ is a summability set for $u$ and $v$ in $[a, b]$ and $k_{i}$ is a number such that if $G$ is $G_{i}$ and $k$ is $k_{i}$ then (2.1) is true. Then $k_{1}=k_{2}$.

Proof. To prove the lemma, we show that if $\epsilon>0$ then $\left|k_{1}-k_{2}\right|$ $<6 \epsilon$. Suppose that $\epsilon>0$; for $i=1,2$, let $A_{i}$ denote a subdivision of $[a, b]$, each of whose terms is in $G_{i}$, such that if $E_{i}$ is a refinement of $A_{i}$ each of whose terms is in $G_{i}$ then $\left|S_{E}(i u, v)-k_{i}\right|<\epsilon$ and $\left|S_{A_{i}}(u, v)-S_{E_{i}}(u, v)\right|<\epsilon$. Let $A$ denote the subdivision of $[a, b]$ whose terms are the terms of $A_{1}$ and $A_{2}$.

Case I; each of the terms of $A$ is in $G_{1}$ and in $G_{2}$. In this case, $\left|S_{A}(u, v)-k_{1}\right|<\epsilon$ and $\left|S_{A}(u, v)-k_{2}\right|<\epsilon$; so $\left|k_{1}-k_{2}\right|<2 \epsilon<6 \epsilon$.

Case II; there is a term of $A$ which is not in $G_{1}$ or not in $G_{2}$. Let 
$x_{1}, x_{2}, \cdots, x_{n}$ denote the terms of $A$ which are not in $G_{1}$ or not in $G_{2}$. For $p=1,2, \cdots, n$, let $\left[c_{p}, d_{p}\right]$ denote a subinterval of $[a, b]$ and $C_{p}$ a subdivision of $\left[c_{p}, d_{p}\right]$ such that

(i) each of $c_{p}$ and $d_{p}$ is in $G_{1}$ and in $G_{2}$,

(ii) $c_{p}<x_{p}<d_{p}$, and

(iii) if $D_{p}$ is a refinement of $C_{p}$ then $\left|S_{C_{p}}(u, v)-S_{D_{p}}(u, v)\right|<\epsilon / n$. Let $D$ denote the subdivision of $[a, b]$ whose terms are the terms of $C_{1}, C_{2}, \cdots, C_{n}$, and $A$. If each of the terms of $D$ is in $G_{1}$, then $\left|S_{D}(u, v)-k_{1}\right|<\epsilon<3 \epsilon$. Suppose that there is a term of $D$ which is not in $G_{1}$; let $t_{1}, t_{2}, \cdots, t_{m}$ denote the terms of $D$ which are not in $G_{1}$. If $k$ is one of the first $m$ positive integers, then there is an integer $p$ such that $c_{p}<t_{k}<d_{p}$, and (by Definition 2.1 and Lemma 2.2) there is a subinterval $\left[r_{k}, s_{k}\right]$ of $\left[c_{p}, d_{p}\right]$ such that $r_{k}$ and $s_{k}$ are in $G_{1}$, and $r_{k}<t_{k}<s_{k}$, and $\left|S_{K}(u, v)-S_{L}(u, v)\right|<\epsilon / 2 m$ if $K$ and $L$ are subdivisions of $\left[r_{k}, s_{k}\right]$. Let $E$ denote the refinement of $D$ whose terms are the terms of $D$ and the numbers $r_{k}$ and $s_{k}, k=1,2, \cdots, m$; then $\left|S_{D}(u, v)-S_{E}(u, v)\right|<\epsilon$. Let $F$ denote the subdivision of $[a, b]$ whose terms are the terms of $E$ which are in $G_{1}$; then $\left|S_{E}(u, v)-S_{F}(u, v)\right|$ $<\epsilon$. Moreover, $F$ is a refinement of $A_{1}$ each of whose terms is in $G_{1}$; so $\left|S_{F}(u, v)-k_{1}\right|<\epsilon$. Hence $\left|S_{D}(u, v)-k_{1}\right|<3 \epsilon$. By a similar argument, $\left|S_{D}(u, v)-k_{2}\right|<3 \epsilon$; so $\left|k_{1}-k_{2}\right|<6 \epsilon$. This completes the proof.

REMARK 2.2. If $u$ is integrable with respect to $v$ in $[a, b]$, then $[a, b]$ is a summability set for $u$ and $v$ in $[a, b]$, and $\int_{a}^{b} u(x) d v(x)$ is the number $k$ of Lemma 2.1 .

Definition 2.4. Suppose that $u$ is a function, $v$ is a function, and $[a, b]$ is an interval.

(i) The statement that $u$ is summable with respect to $v$ in $[a, b]$ means that there is a summability set for $u$ and $v$ in $[a, b]$.

(ii) if $u$ is summable with respect to $v$ in $[a, b]$, then $\int_{a}^{b} u(x) d v(x)$, the integral of $u$ with respect to $v$ in $[a, b]$, is the number $k$ of Lemma 2.1 , and $\int_{b}^{a} u(x) d v(x)=-\int_{a}^{b} u(x) d v(x)$.

3. Some properties of the integral. We shall now show that the

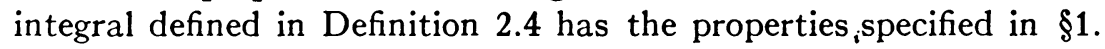
It follows directly from Definition 2.4 that the statement (1.1) is true if $X$ denotes the subset of $U$ such that a member $(v, u,[a, b])$ of $U$ is in $X$ if and only if $u$ is summable with respect to $v$ in $[a, b]$.

THEOREM 3.1. Suppose that the function $u$ is summable with respect to the function $v$ in the interval $[a, b]$, and $a_{11}, a_{12}, a_{13}, a_{21}, a_{22}, a_{23}$ is a number seguence, and $f(x)=a_{11} u(x)+a_{12} v(x)+a_{13}$ and $g(x)=a_{21} u(x)$ $+a_{22} v(x)+a_{23}$ for each number $x$ in $[a, b]$. Then $f$ is summable with respect to $g$ in $[a, b]$, and 


$$
\begin{aligned}
& \int_{a}^{b} f(x) d g(x)-2^{-1}[f(a)+f(b)][g(b)-g(a)] \\
& =\left(a_{11} a_{22}-a_{12} a_{21}\right)\left\{\int_{a}^{b} u(x) d v(x)-2^{-1}[u(a)+u(b)][v(b)-v(a)]\right\} .
\end{aligned}
$$

Proof. If $D$ is a subdivision of a subinterval $[c, d]$ of $[a, b]$, then

$$
\begin{aligned}
S_{D}(f, g)= & a_{21} S_{D}(f, u)+a_{22} S_{D}(f, v) \\
= & a_{11} a_{21} S_{D}(u, u)+a_{12} a_{21} S_{D}(v, u)+a_{13} a_{21} S_{D}(1, u) \\
& +a_{11} a_{22} S_{D}(u, v)+a_{12} a_{22} S_{D}(v, v)+a_{13} a_{22} S_{D}(1, v) \\
= & \left.2^{-1} a_{11} a_{21} u^{2}\right|_{c} ^{d}+\left.a_{12} a_{21} u v\right|_{c} ^{d}-a_{12} a_{21} S_{D}(u, v)+\left.a_{13} a_{21} u\right|_{c} ^{d} \\
& +a_{11} a_{22} S_{D}(u, v)+\left.2^{-1} a_{12} a_{22} v^{2}\right|_{c} ^{d}+\left.a_{13} a_{22} v\right|_{c} ^{d} .
\end{aligned}
$$

Hence if $D$ and $E$ are subdivisions of $[c, d]$, then

$$
S_{D}(f, g)-S_{E}(f, g)=\left(a_{11} a_{22}-a_{12} a_{21}\right)\left[S_{D}(u, v)-S_{E}(u, v)\right] .
$$

Hence if $G$ is a summability set for $u$ and $v$ in $[a, b]$, then $G$ is a summability set for $f$ and $g$ in $[a, b]$. Moreover, if $D$ is a subdivision of $[a, b]$ and $E$ is the subdivision of $[a, b]$ whose only terms are $a$ and $b$, then

$$
\begin{aligned}
S_{D}(f, g) & -2^{-1}[f(a)+f(b)][g(b)-g(a)] \\
& =\left(a_{11} a_{22}-a_{12} a_{21}\right)\left\{S_{D}(u, v)-2^{-1}[u(a)+u(b)][v(b)-v(a)]\right\} .
\end{aligned}
$$

The theorem now follows at once.

COROLlaRY 3.1a. If $u$ is summable with respect to $v$ in $[a, b]$, then $v$ is summable with respect to $u$ in $[a, b]$, and $\int_{a}^{b} u(x) d v(x)=u(b) v(b)$ $-u(a) v(a)-\int_{a}^{b} v(x) d u(x)$.

LEMMA 3.2a. If the function $u$ is summable with respect to the function $v$ in the interval $[a, b]$ and $x$ is a number in $[a, b]$, then $x$ is not an exceptional number for $u$ and $v$ in $[a, b]$.

Proof. Let $G$ denote a summability set for $u$ and $v$ in $[a, b]$. If $x$ is in $G$, then by Definition 2.3, $x$ is not an exceptional number for $u$ and $v$ in $[a, b]$. Suppose that $x$ is not in $G$; let $H$ denote the complement of $G$ in $[a, b]$; then $x$ is in $H$, and $(v, u, H)$ is a singular graph. Let $\epsilon$ denote a positive number. Then there is a subinterval $\left[c_{1}, d_{1}\right]$ of $[a, b]$ such that $c_{1}$ and $d_{1}$ are in $G, x$ is between $c_{1}$ and $d_{1}$, and 
$\left|S_{C_{1}}(u, v)-S_{D_{1}}(u, v)\right|<\epsilon / 2$ if $C_{1}$ and $D_{1}$ are subdivisions of $\left[c_{1}, d_{1}\right]$. Suppose that there is a subinterval $[c, d]$ of $[a, b]$ containing $x$ such that if $[p, q]$ is a subinterval of $[c, d]$ which does not contain $x$ then $u$ is integrable with respect to $v$ in $[p, q]$. If $c \leqq x<d$, let $p$ denote a number less than $d$ between $x$ and $d_{1}$, and let $C_{2}$ denote a subdivision of $[p, d]$ such that $\left|S_{C_{2}}(u, v)-S_{D_{2}}(u, v)\right|<\epsilon / 2$ if $D_{2}$ is a refinement of $C_{2}$, and such that $d_{1}$ is a term of $C_{2}$ if $d_{1}<d$. By Lemma 2.2, there is a subdivision $C_{3}$ of $[x, p]$ such that if $D_{3}$ is a refinement of $C_{3}$ then $\left|S_{C_{3}}(u, v)-S_{D_{3}}(u, v)\right|<\epsilon / 2$; hence it follows that there is a subdivision $C$ of $[x, d]$ such that if $D$ is a refinement of $C$ then $\left|S_{C}(u, v)-S_{D}(u, v)\right|<\epsilon$; so $u$ is integrable with respect to $v$ in $[x, d]$. Similarly, if $c<x \leqq d$, then $u$ is integrable with respect to $v$ in $[c, x]$. Hence $u$ is integrable with respect to $v$ in $[c, d]$ and consequently in each subinterval of $[c, d]$; so $x$ is not an exceptional number for $u$ and $v$ in $[a, b]$. This completes the proof.

THEOREM 3.2. Suppose that $u$ is summable with respect to $v$ in $[a, b]$, and that $a<c<b$. Then $u$ is summable with respect to $v$ in $[a, c]$ and in $[c, b] ;$ and $\int_{a}^{c} u(x) d v(x)+\int_{c}^{b} u(x) d v(x)=\int_{a}^{b} u(x) d v(x)$.

Proof. Let $G$ denote a summability set for $u$ and $v$ in $[a, b]$, let $G_{1}$ denote the set whose members are $c$ and the members of $G$ which are in $[a, c]$, and let $G_{2}$ denote the set whose members are $c$ and the members of $G$ which are in $[c, b]$. If $c$ is in $G$, it readily follows from Definition 2.3 and Lemma 2.2 that $G_{1}$ and $G_{2}$ are summability sets for $u$ and $v$ in $[a, c]$ and $[c, b]$, respectively, and consequently that the conclusion of the theorem is true. Suppose that $c$ is not in $G$. By Lemma 3.2a, $c$ is not an exceptional number for $u$ and $v$ in $[a, b]$ and hence is not an exceptional number for $u$ and $v$ in $[a, c]$ or in $[c, b]$. Since $c$ is not in $G$, it follows that if $\epsilon$ is a positive number, then there is a subinterval $[p, q]$ of $[a, b]$ such that $p$ and $q$ are in $G, c$ is between $p$ and $q$, and $\left|S_{P}(u, v)-S_{Q}(u, v)\right|<\epsilon$ if $P$ and $Q$ are subdivisions of $[p, q]$. From Lemma 2.2 and Definition 2.3 it readily follows that $G_{1}$ and $G_{2}$ are summability sets for $u$ and $v$ in $[a, c]$ and $[c, b]$, respectively, and consequently that the conclusion of the theorem is true. This completes the proof.

TheOREM 3.3. Suppose that $[a, b]$ is an interval, $H$ is a subset of the segment $(a, b), G$ is the complement of $H$ in $[a, b]$, and $u, v, u_{1}, v_{1}$ are functions such that

(i) $u_{1}$ is integrable with respect to $v_{1}$ in $[a, b]$,

(ii) each of $(v, u, H)$ and $\left(v_{1}, u_{1}, H\right)$ is a singular graph,

(iii) $u(x)=u_{1}(x)$ and $v(x)=v_{1}(x)$ if $x$ is in $G$, and 
(iv) if $x$ is in $G$ then $x$ is not an exceptional number for $u$ and $v$ in $[a, b]$.

Then $G$ is a summability set for $u$ and $v$ in $[a, b]$, and $f_{a}^{b} u(x) d v(x)=$ $\int_{a}^{b} u_{1}(x) d v_{1}(x)$.

Proof. By hypothesis $u_{1}$ is integrable with respect to $v_{1}$ in $[a, b]$; so if $x$ is in $G$, then $x$ is not an exceptional number for $u_{1}$ and $v_{1}$ in $[a, b]$, and since $\left(v_{1}, u_{1}, H\right)$ is a singular graph it can readily be seen that $G$ is a summability set for $u_{1}$ and $v_{1}$ in $[a, b]$. But if $D$ is a subdivision of $[a, b]$ each of whose terms is in $G$, then $S_{D}(u, v)=S_{D}\left(u_{1}, v_{1}\right)$, and consequently $G$ is a summability set for $u$ and $v$ in $[a, b]$ and $\int_{a}^{b} u(x) d v(x)=\int_{a}^{b} u_{1}(x) d v_{1}(x)$. This completes the proof.

\section{REFERENCES}

1. R. E. Lane, The integral of a function with respect to a function, Proc. Amer. Math. Soc. vol. 5 (1954) pp. 59-66.

2. H. L. Smith, On the existence of the Stieltjes integral, Trans. Amer. Math. Soc. vol. 27 (1925) pp. 491-515.

3. H. S. Kaltenborn, Existence conditions and a substitution theorem for Stieltjes mean integrals, Tôhoku Math. J. vol. 44 (1938) pp. 1-11.

The University of Texas 